\title{
The reliability of neuroanatomy as a predictor of eloquence: a review
}

\author{
Nader Pouratian, M.D., Ph.D., ${ }^{1}$ And Susan Y. Bookheimer, Рh.D. ${ }^{2,3}$ \\ Departments of ${ }^{1}$ Neurosurgery, ${ }^{2}$ Psychiatry and Biobehavioral Science, and ${ }^{3}$ Psychology, \\ David Geffen School of Medicine at UCLA, Los Angeles, California
}

\begin{abstract}
The adjacency of intracranial pathology to canonical regions of eloquence has long been considered a significant source of potential morbidity in the neurosurgical care of patients. Yet, several reports exist of patients who undergo resection of gliomas or other intracranial pathology in eloquent regions without adverse effects. This raises the question of whether anatomical and intracranial location can or should be used as a means of estimating eloquence. In this review, the authors systematically evaluate the factors that are known to affect anatomical-functional relationships, including anatomical, functional, pathology-related, and modality-specific sources of variability. This review highlights the unpredictability of functional eloquence based on anatomical features alone and the fact that patients should not be considered ineligible for surgical intervention based on anatomical considerations alone. Rather, neurosurgeons need to take advantage of modern technology and mapping techniques to create individualized maps and management plans. An individualized approach allows one to expand the number of patients who are considered for and who potentially may benefit from surgical intervention. Perhaps most importantly, an individualized approach to mapping patients with brain tumors ensures that the risk of iatrogenic functional injury is minimized while maximizing the extent of resection. (DOI: 10.3171/2009.11.FOCUS09239)
\end{abstract}

KEY WoRDS • brain mapping •
functional magnetic resonance imaging

$\mathrm{T}$ HE adjacency of intracranial pathology to canonical regions of eloquence has long been considered a significant source of potential morbidity in the neurosurgical care of patients. ${ }^{52}$ Yet, several reports exist of patients who undergo resection of gliomas or other intracranial pathology in eloquent regions without adverse effects. ${ }^{37,39}$ For example, Plaza and colleagues ${ }^{37}$ recently reported on a patient who underwent resection of a posteroinferior left frontal glioma. The patient's speech was grossly intact even after resection of the Broca area. Such reports not only highlight the "efficiency of brain plasticity," but also call into question whether anatomical and intracranial location can or should be used as a means of estimating eloquence. It is increasingly apparent from the scientific and clinical literature that the relationship between anatomy and function is not as clearly coupled as once believed and that this relationship is even more complicated in patients with intracranial pathology. ${ }^{9}$

The sources of variability of functional localization, including anatomical, functional, pathology-related, and modality-specific sources, are systematically reviewed in this manuscript, highlighting the unpredictability of

Abbreviations used in this paper: AVM = arteriovenous malformation; $\mathrm{ECoG}$ = electrocorticography; $\mathrm{ESM}$ = electrocortical stimulation mapping; $\mathrm{LGG}=$ low-grade glioma. functional eloquence based on anatomical features alone. We argue that patients should not be considered ineligible for surgical intervention based on anatomical considerations alone. Rather, neurosurgeons need to take advantage of modern technology and mapping techniques to create individualized maps and management plans for each patient.

\section{Anatomical Variability}

Across patients, the human brain has a stereotypical pattern of gyri and sulci that forms the basis of neuroanatomical teaching. In patients without intracranial mass lesions, the consistent patterns of sulci and gyri enable clinicians and researchers to use neuroimaging studies to reliably identify neuroanatomical structures. For example, Kido and colleagues ${ }^{25}$ identified a constant relationship between the posterior end of the superior frontal sulcus and the precentral sulcus to aid in the localization of the precentral gyrus. Similarly, Yousry and colleagues ${ }^{60}$ suggested the precentral gyrus (even more specifically, the hand region of the motor strip) can be identified by the presence of a "knob-like" structure that is shaped like the Greek letters omega or epsilon on axial imaging. These common features across patients at least partially justify the development of neuroanatomical atlases such 
as those of Talairach and Tournoux, and Schaltenbrand and Wahren, and the common practice in neuroscientific research to refer to specific cerebral areas by coordinates (for example, in the Talairach coordinate system, the inferior frontal gyrus corresponds to $-56,+12,+32) .46,54$

Despite these common features and neurosurgery's historical reliance on atlases, there is tremendous interpatient neuroanatomical variability. Single-patient atlases, such as that of Schaltenbrand and Wahren, cannot account for normal variability across patients, let alone more complex variability introduced by aging, disease, and other unanticipated factors. Technological advances and increased availability of neuroimaging have made it easier to recognize this variability across patients. For example, Caulo and colleagues ${ }^{13}$ reassessed the "knoblike" region of the motor strip in 257 patients and found 5 variants of the regions, 3 of which were previously undescribed. Likewise, Ebeling and colleagues ${ }^{19}$ characterized the inferior precentral sulcus anatomy and identified 4 major anatomical variants. Anatomical variability is perhaps best highlighted by average intensity volumetric atlases, such as the MNI 305 and ICBM 452, which use multiparameter transformations and high-order polynomial warping to align brains of multiple patients, but still contain indistinct representations of both cortical and subcortical structures (Fig. 1). ${ }^{47}$ Because of this, Shattuck and colleagues ${ }^{47}$ constructed a probabilistic atlas in which they provided a voxel-by-voxel probability of finding a specific cortical structure at a given location, demonstrating a high degree of variability in the spatial localization of each cortical landmark. Similar probabilistic anatomical maps have been generated for subcortical structures. ${ }^{1}$ Neuroanatomical studies have identified multiple factors that affect anatomical variability, including but not limited to sex, handedness, aging, and neurological and psychiatric diseases such as depression, psychosis, and dementia. $2,3,30,33,56,57$

In the neurosurgical population, additional interpatient anatomical variability arises from the presence of intracranial pathology. Space-occupying lesions can efface adjacent sulci such that normal anatomical and imaging landmarks are more difficult or impossible to identify ${ }^{42,59}$ Moreover, slow-growing low-grade tumors can infiltrate (and widen) neighboring gyri in such a way as to obliterate normal anatomical landmarks but retain function within the limits of the tumor. ${ }^{49}$

Interpreting the relationship between anatomy and function and the significance of neuroanatomical variability is further complicated by the fact that anatomical variability is associated with and may itself contribute to distinct patterns of functional organization. For example, interhemispheric anatomical asymmetries (especially with respect to the planum temporale) have repeatedly been shown to be related to language lateralization (see Josse and Tzourio-Mazoyer ${ }^{24}$ for review). ${ }^{23,53}$ These studies unambiguously demonstrate that anatomical variability and functional organization are intimately related in multiple dimensions, in ways that are difficult to account for on an individual basis.

Given current technology and paradigms, the potential factors that can affect neuroanatomical variability,
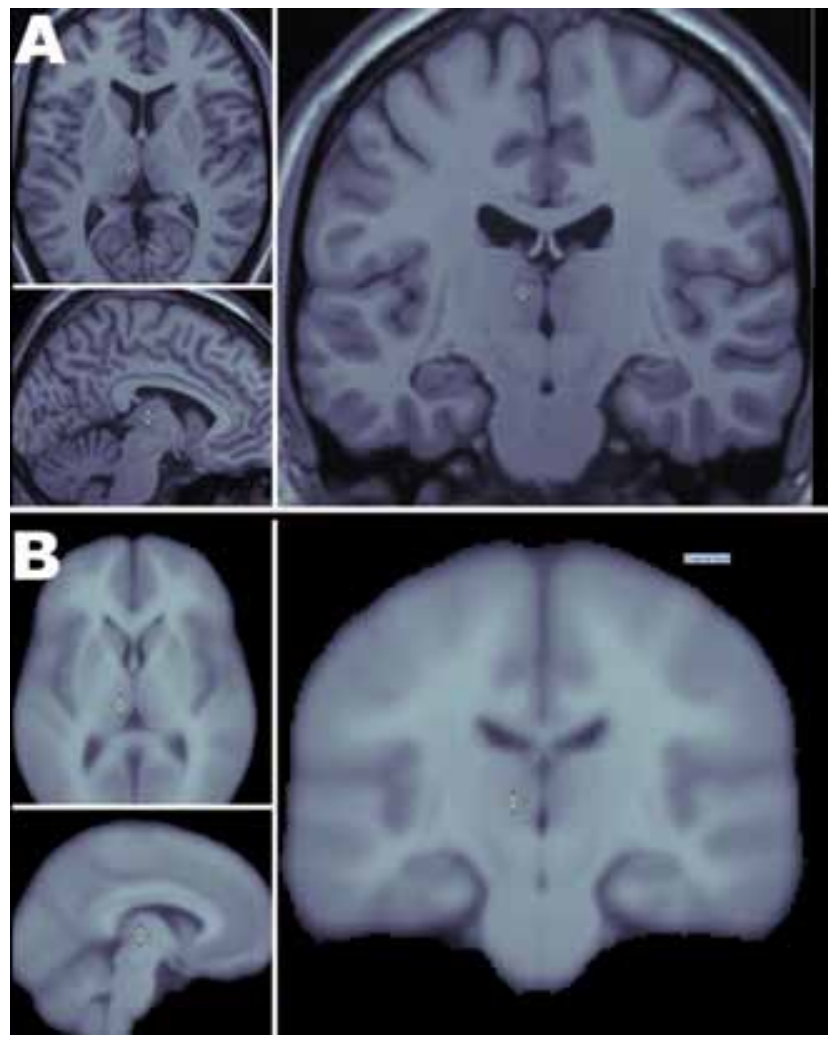

FIG. 1. Anatomical variability of cerebral anatomy across 452 patients (ICBM 452 Atlas). A: Axial, sagittal, and coronal views of a T1-weighted MR image of a single patient's brain, demonstrating the high degree of precision with which a single patient's cerebral anatomy can be imaged. B: Axial, sagittal, and coronal views of the average brain of 452 patients who were imaged using the same imaging protocol as that in $\mathbf{A}$. In this "average brain," patients' brains were linearly transformed into a common space using a 12-parameter affine transformation.

particularly the unpredictable effect of intracranial mass lesions on neighboring brain, are likely too numerous to account for and make it difficult to use neuroanatomical landmarks to predict eloquence when evaluating an individual's brain.

\section{Intrinsic Functional Variability}

Early reports of functional organization suggested that function is tightly coupled to anatomy and that anatomical and imaging landmarks can be reliably used to predict the functional organization of the brain. ${ }^{4,25}$ For example, in the report described above by Kido and colleagues, ${ }^{25}$ the authors suggest that the knob-like landmark of the precentral gyrus was highly predictive of the primary motor cortex dedicated to control of the hand. The assumption of tight and specific functional-anatomical coupling emerged in part from Penfield's depiction of the homunculus, which was intended as a pictorial simplification of his original findings.

Over time, it has become increasingly apparent that even with anatomical constraints to account for structural variability, functional localization is highly variable.,20,48,55 For example, the concept of the precentral knob representing the primary motor area of the hand has 


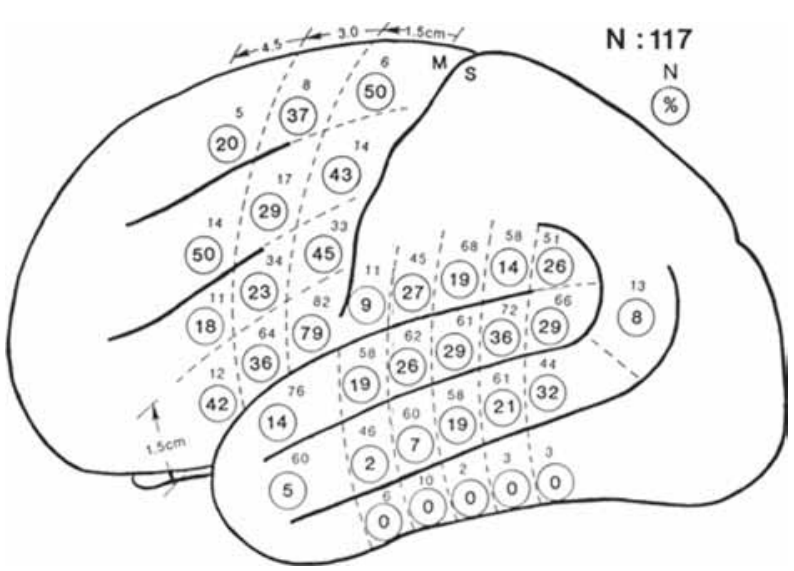

FIG. 2. Image demonstrating the variability in language localization in 117 patients. Electrocortical stimulation maps from 117 patients were plotted in a common space, based on major anatomical landmarks. The upper number in each area is the number of patients who had language testing performed in that cortical region and the lower number (in the circle) represents the percentage of all patients tested in that cortical region in whom cortical stimulation evoked significant naming errors (essential language sites). $M=$ motor cortex; $S=$ sensory cortex. From Ojemann et al:: Cortical language localization in left, dominant hemisphere. An electrical stimulation mapping investigation in 117 patients. J Neurosurg 71:316-326, 1989.

been called into question with a recent report suggesting that depending on the patient, the "knob" can either represent primary motor or premotor cortex. ${ }^{48}$ Others have reported variability in the functional organization of the primary sensorimotor cortices as well. For example, within the precentral gyrus, stimulation of individual cortical sites has been shown to recruit both sensory and motor phenomenon and in other cases stimulation has been shown to recruit motor movements in more than 1 motor group. ${ }^{7,20,45}$ Variability in essential language cortices has also been described. For example, even after accounting for the anatomical variability of the posterior inferior frontal area, the University of California San Francisco group reported $>4 \mathrm{~cm}$ of variability in the localization of speech arrest sites in that region. ${ }^{42}$

Although the University of California San Francisco group studied the variability of language function within anatomical constraints, the representation and variability of language cortices is generally more widespread, involving multiple cortical regions throughout the frontal, parietal, and temporal lobes..$^{17,34,44}$ In fact, the distribution of language sites is so vast that it is easier to predict where language will not be (for example, the inferior temporal gyrus) than where it is likely to be..$^{34}$ Not all areas, however, are equally likely to be essential for language function. For example, Ojemann and colleagues ${ }^{34}$ reported that the posterior inferior frontal region is essential in $79 \%$ of patients, whereas the anterior middle temporal gyrus is essential in only $5 \%$ of patients (Fig. 2). The variability of speech arrest sites in the frontal lobe (the Broca area) is generally greater than that of the parietotemporal regions (the Wernicke area). Despite the widespread distribution at a population level, essential language sites in an individual are usually discreet and occupy a smaller surface area than traditional descriptions of the Broca and Wer- nicke areas. ${ }^{34}$ These ESM studies of functional variability have important limitations. Although intrinsic functional variability likely contributes significantly to the reported results, the effect of intracranial pathology (such as epilepsy and tumor) on the reported functional variability cannot be excluded or separated (discussed below). Also, these studies divide the brain into parcels based on predetermined metric distances $(1$ or $3 \mathrm{~cm}$ ) rather than anatomical landmarks and therefore do not and cannot wholly account for the contribution of anatomical variability or intracranial mass lesions to the final results.

\section{Disease-Related Functional Variability}

The anatomical and functional variability described above are complex enough to make it unlikely to predict eloquence with a high level of precision and accuracy even in healthy patients. These intrinsic sources of variability, which are present in every individual, are compounded by the presence of intracranial pathology. Besides changes in cerebral anatomy (discussed above), intracranial pathology can introduce at least 3 additional sources of potential variability.

First, the presence of intracranial lesions, especially congenital lesions such as AVMs, may result in distinct patterns of functional acquisition. For example, several studies have noted a greater preponderance of right-sided language lateralization in patients with cerebrovascular malformations. ${ }^{28,58} \mathrm{By}$ comparing these patients with those with adult-onset left hemisphere brain injury, who retain a normal distribution of language lateralization, Vikingstad and colleagues ${ }^{58}$ suggested that the congenital nature of vascular malformations contributes to the atypical patterns of functional acquisition. Likewise, in patients with left temporal lobe epilepsy, earlier age of onset has been associated with a greater likelihood of atypical language lateralization, suggesting that the presence of intracranial pathology may affect acquisition (or consolidation) patterns. ${ }^{8,22}$ Whether these case series represent differential acquisition as opposed to reorganization is not entirely clear and is subject of great debate. However, for the purposes of the current discussion, the definitive cause for atypical organization is not necessarily important. What these and other studies show is that functional organization patterns (especially that of language) are different in patients with intracranial pathology and healthy patients.

As noted, intracranial pathology may not only affect acquisition patterns, but can also induce functional reorganization, in which functional representations shift to adjacent or remote (contralateral) regions due to insult to or progressive enlargement or involvement of eloquent structures, such as may be the case with LGGs. For example, Lucas and colleagues ${ }^{29}$ compared language maps in patients with adult-acquired neurological injury (including glioma, subarachnoid hemorrhage, and traumatic brain injury) with age-matched controls and found that patients with neurological injuries have a greater proportion of frontal lobe language areas than controls, suggesting functional reorganization of language representations in these patients. The degree of functional reorganization and compensation, both neurophysiologically and 
behaviorally, is likely related to the time course of the disease, a topic reviewed extensively by Desmurget and colleagues. ${ }^{16}$ This relationship is perhaps best exemplified by the fact that patients with LGG rarely present with neurological deficits. ${ }^{36}$ Moreover, mapping studies in patients with LGG demonstrate multiple patterns of reorganization and compensation, including identifying function persisting within the tumor or redistributed immediately around the tumor, throughout the ipsilateral hemisphere, or into the contralateral hemisphere. ${ }^{16}$ In all cases, patients seemingly have no deficit. This result is in stark contrast to outcomes in patients with acute stroke, who do not compensate or recover as well as patients with LGG of the same size and in the same location..$^{16}$ Studies of serial intraoperative mapping in patients with repeated surgeries for gliomas unambiguously demonstrated that the functional organization of the brain is dynamic and is subject to acute changes in the local environment. For example, Robles and colleagues ${ }^{43}$ reported on 2 patients in whom maps of eloquent language cortices changed between surgeries spaced by several years, allowing a multistaged surgical approach for resection of LGGs in eloquent cortices. Duffau and colleagues ${ }^{18}$ have also similarly reported acute patterns of reorganization during the same surgery, again reinforcing the concept that short- and long-term changes in the intracranial environment can lead to reorganization or unmasking of eloquent functions that would be impossible to predict based on anatomical localization alone.

Besides influencing the functional organization of the brain, intracranial disease can also cause disease-related imaging artifacts. Although this characteristic does not specifically affect the relationship between anatomy and function, it introduces yet another level of uncertainty in assessing eloquence. For example, some argue that functional MR imaging cannot be used to map eloquent cortices adjacent to AVMs because AVMs may alter the perfusion-dependent response that functional MR imaging relies on or because AVMs cause susceptibility artifacts that can interfere with detection of the blood oxygen level-dependent functional MR imaging response. These potentially disease-specific preoperative mapping limitations suggest that it is important to verify the reliability and accuracy of different mapping modalities in each disease population. For example, we specifically tested the accuracy and reliability of blood oxygen level-dependent functional MR imaging mapping in patients with vascular malformations and found that functional MR imaging is highly sensitive and specific for determining language localization in patients with vascular malformations, even directly adjacent to these lesions. ${ }^{39}$

\section{Mapping-Related Sources of Variability in Functional Localization}

The myriad of preoperative and intraoperative functional brain mapping techniques raise yet another layer of complexity. Although all mapping modalities measure functional changes that are related to underlying neuronal activity, the maps generated by various mapping techniques may not be directly comparable because the pre- cise signal measured with each technique differs. Moreover, despite extensive research, the precise significance and relationship between various mapping signals is not necessarily well understood. Broadly speaking, functional brain mapping techniques localize brain function by measuring either electrophysiological or perfusion-related signals. Electrophysiological maps can be generated using single unit recordings, ECoG and local field potentials, and electroencephalographic or magnetoencephalographic techniques. On the other hand, PET, functional MR imaging, and optical imaging generate maps based on perfusion-related signals that are coupled to neuronal activity (to support local metabolism). Cortical stimulation mapping and transcranial magnetic stimulation are in a class of their own because maps are generated by inducing temporary lesions to disrupt function rather than measuring activity-related signals.

While all mapping techniques will (or should) produce similar maps, intermodality variation exists due to differences in techniques and underlying assumptions. The relationships between these various mapping techniques have been the subject of numerous studies, including comparisons between ESM and PET, ${ }^{6}$ ESM with functional MR imaging, ${ }^{39} \mathrm{ECoG}$ and $\mathrm{ESM},{ }^{10} \mathrm{ECoG}$ with functional MR imaging, ${ }^{26,32}$ optical imaging and functional MR imaging, ${ }^{11,38,41}$ electroencephalography with functional MR imaging, ${ }^{31}$ and functional MR imaging with single units and local field potentials. ${ }^{32,35}$ Ultimately, for clinical relevance and implementation, each technique will have to be evaluated relative to patient outcomes. In the meantime, the most important comparisons from a clinical perspective are those between each technique and ESM. Whereas most mapping techniques identify all areas that are recruited by a specific task (detecting all activity-related signals), ESM only identifies essential areas, or areas without which the task cannot be executed. Consequently, in most such comparisons, techniques that map nonessential cortices are considered to be very sensitive but not very specific. With current technologies and implementation, preoperative and intraoperative mapping techniques besides ESM at best provide a framework within which to map the exposed brain but cannot replace ESM. ${ }^{10,39}$ Further discussion of studies comparing the various mapping signals is beyond the scope of this review but are critical for gaining insight into the physiological basis of and the relationship between the different modalities. The salient point is that while all signals are related, there are inherent differences in modalityspecific maps that must be considered.

In addition to intermodality variability, one must also consider trial-to-trial variability of mapping signals (which can result in distinct functional maps) and variability of functional maps related to differences in technique and analysis. ${ }^{15,40}$ For most mapping modalities, post hoc analysis of acquired data are as important, if not more important, than the original acquisition parameters. The sources of variability can be center-specific and therefore may change the reliability, accuracy, or clinical relevance of one mapping technique from one medical center to another. 


\section{Conclusions}

Despite a tradition in neurosurgery of relying on standardized atlases and anatomically defined regions of eloquence to guide surgical decision making, we must instead rely on individualized data and maps that can account for much (but not all) of the variability discussed. Moreover, we must rely on mapping techniques that have been validated relative to patient outcomes, rather than relative to a surrogate marker. Because we are practicing in an era in which neuroimaging, functional imaging, and intraoperative brain mapping are more accessible than ever, it is imperative that lesions in or near eloquent regions are managed using an individualized approach. An individualized approach allows us to make informed clinical and operative recommendations for each patient ${ }^{12}$ and expands the number of patients who are considered for and who potentially may benefit from surgical intervention.

Given the probability that more extensive resection of gliomas is associated with improved outcomes, ${ }^{21,50}$ all patients with gliomas should be given serious consideration for resective surgery. Resectability is affected by multiple factors, including size, diffuse borders, insular or temporal lobe involvement, and presumed eloquent location. ${ }^{14}$ When eloquent location is one of the primary factors limiting consideration for resection, further preoperative functional evaluation is mandatory. Neurosurgeons practicing in facilities that do not have means for preoperative brain mapping (functional MR imaging) or intraoperative stimulation mapping should refer such patients to centers that can offer these services. There is no "gold standard' for preoperative mapping and evaluation because no preoperative brain mapping technique has been validated with respect to outcomes. Nonetheless, based on our experience, preoperative evaluation should include functional MR imaging at a minimum. Evaluation of resectability may be augmented with addition of diffusion tensor imaging to visualize the position and integrity of white matter tracts deep to and around the lesion.,27 Alternative approaches that have been advocated and could be considered include PET and magnetoencephalography/magnetic source imaging, depending on the experience, expertise, and availability of technology at a particular center. ${ }^{5,51}$ Regardless of technique used, preoperative maps should be evaluated by a neurosurgeon who is experienced in interpreting these maps to determine the best surgical approach. In patients in whom resection is attempted, intraoperative stimulation mapping is mandatory because it is the only technique with validated outcome measures. ${ }^{34,44}$

An individualized approach to mapping patients with brain tumors such as the one described in this paper will ensure that the risk of iatrogenic functional injury is minimized while maximizing the extent of resection. ${ }^{43}$

\section{Disclosure}

The authors report no conflict of interest concerning the materials or methods used in this study or the findings specified in this paper.

Author contributions to the study and manuscript prepara- tion include the following: Drafting the article: N Pouratian, SY Bookheimer. Critically revising the article: N Pouratian, SY Bookheimer. Reviewed final version of the manuscript and approved it for submission: N Pouratian, SY Bookheimer.

\section{Acknowledgment}

The authors thank Ivo Dinov, Ph.D., of the UCLA Laboratory of Neuro Imaging, for providing some of the components of Fig. 3.

\section{References}

1. Ahsan RL, Allom R, Gousias IS, Habib H, Turkheimer FE, Free S, et al: Volumes, spatial extents and a probabilistic atlas of the human basal ganglia and thalamus. Neuroimage 38:261-270, 2007

2. Annett M: Parallels between asymmetries of planum temporale and of hand skill. Neuropsychologia 30:951-962, 1992

3. Ballmaier M, Sowell ER, Thompson PM, Kumar A, Narr KL, Lavretsky $\mathrm{H}$, et al: Mapping brain size and cortical gray matter changes in elderly depression. Biol Psychiatry 55:382389, 2004

4. Berger MS, Cohen WA, Ojemann GA: Correlation of motor cortex brain mapping data with magnetic resonance imaging. J Neurosurg 72:383-387, 1990

5. Berman JI, Berger MS, Chung SW, Nagarajan SS, Henry RG: Accuracy of diffusion tensor magnetic resonance imaging tractography assessed using intraoperative subcortical stimulation mapping and magnetic source imaging. J Neurosurg 107:488-494, 2007

6. Bookheimer SY, Zeffiro TA, Blaxton T, Malow BA, Gaillard WD, Sato S, et al: A direct comparison of PET activation and electrocortical stimulation mapping for language localization. Neurology 48:1056-1065, 1997

7. Branco DM, Coelho TM, Branco BM, Schmidt L, Calcagnotto ME, Portuguez M, et al: Functional variability of the human cortical motor map: electrical stimulation findings in perirolandic epilepsy surgery. J Clin Neurophysiol 20:17-25, 2003

8. Brázdil M, Chlebus P, Mikl M, Pazourková M, Krupa P, Rektor I: Reorganization of language-related neuronal networks in patients with left temporal lobe epilepsy-an fMRI study. Eur J Neurol 12:268-275, 2005

9. Brett M, Johnsrude IS, Owen AM: The problem of functional localization in the human brain. Nat Rev Neurosci 3:243249, 2002

10. Brunner P, Ritaccio AL, Lynch TM, Emrich JF, Wilson JA, Williams JC, et al: A practical procedure for real-time functional mapping of eloquent cortex using electrocorticographic signals in humans. Epilepsy Behav 15:278-286, 2009

11. Cannestra AF, Pouratian N, Bookheimer SY, Martin NA, Beckerand DP, Toga AW: Temporal spatial differences observed by functional MRI and human intraoperative optical imaging. Cereb Cortex 11:773-782, 2001

12. Cannestra AF, Pouratian N, Forage J, Bookheimer SY, Martin NA, Toga AW: Functional magnetic resonance imaging and optical imaging for dominant-hemisphere perisylvian arteriovenous malformations. Neurosurgery 55:804-814, 2004

13. Caulo M, Briganti C, Mattei PA, Perfetti B, Ferretti A, Romani GL, et al: New morphologic variants of the hand motor cortex as seen with MR imaging in a large study population. AJNR Am J Neuroradiol 28:1480-1485, 2007

14. Chang EF, Smith JS, Chang SM, Lamborn KR, Prados MD, Butowski N, et al: Preoperative prognostic classification system for hemispheric low-grade gliomas in adults. J Neurosurg 109:817-824, 2008

15. Cohen MS, DuBois RM: Stability, repeatability, and the expression of signal magnitude in functional magnetic resonance imaging. J Magn Reson Imaging 10:33-40, 1999 
16. Desmurget M, Bonnetblanc F, Duffau H: Contrasting acute and slow-growing lesions: a new door to brain plasticity. Brain 130:898-914, 2007

17. Duffau H, Capelle L, Sichez J, Faillot T, Abdennour L, Law Koune JD, et al: Intra-operative direct electrical stimulations of the central nervous system: the Salpêtrière experience with 60 patients. Acta Neurochir (Wien) 141:1157-1167, 1999

18. Duffau H, Sichez JP, Lehéricy S: Intraoperative unmasking of brain redundant motor sites during resection of a precentral angioma: evidence using direct cortical stimulation. Ann Neurol 47:132-135, 2000

19. Ebeling U, Steinmetz H, Huang Y, Kahn T: Topography and identification of the inferior precentral sulcus in MR imaging. AJNR Am J Neuroradiol 10:937-942, 1989

20. Farrell DF, Burbank N, Lettich E, Ojemann GA: Individual variation in human motor-sensory (rolandic) cortex. J Clin Neurophysiol 24:286-293, 2007

21. Gorlia T, van den Bent MJ, Hegi ME, Mirimanoff RO, Weller M, Cairncross JG, et al: Nomograms for predicting survival of patients with newly diagnosed glioblastoma: prognostic factor analysis of EORTC and NCIC trial 26981-22981/CE.3. Lancet Oncol 9:29-38, 2008

22. Janszky J, Mertens M, Janszky I, Ebner A, Woermann FG: Left-sided interictal epileptic activity induces shift of language lateralization in temporal lobe epilepsy: an fMRI study. Epilepsia 47:921-927, 2006

23. Josse G, Mazoyer B, Crivello F, Tzourio-Mazoyer N: Left planum temporale: an anatomical marker of left hemispheric specialization for language comprehension. Brain Res Cogn Brain Res 18:1-14, 2003

24. Josse G, Tzourio-Mazoyer N: Hemispheric specialization for language. Brain Res Brain Res Rev 44:1-12, 2004

25. Kido DK, LeMay M, Levinson AW, Benson WE: Computed tomographic localization of the precentral gyrus. Radiology 135:373-377, 1980

26. Lachaux JP, Fonlupt P, Kahane P, Minotti L, Hoffmann D, Bertrand $\mathrm{O}$, et al: Relationship between task-related gamma oscillations and BOLD signal: new insights from combined fMRI and intracranial EEG. Hum Brain Mapp 28:13681375,2007

27. Leclercq D, Duffau H, Delmaire C, Capelle L, Gatignol P, Ducros M, et al: Comparison of diffusion tensor imaging tractography of language tracts and intraoperative subcortical stimulations. J Neurosurg [epub ahead of print], 2009

28. Lehéricy S, Biondi A, Sourour N, Vlaicu M, du Montcel ST, Cohen L, et al: Arteriovenous brain malformations: is functional MR imaging reliable for studying language reorganization in patients? Initial observations. Radiology 223:672682,2002

29. Lucas TH II, Drane DL, Dodrill CB, Ojemann GA: Language reorganization in aphasics: an electrical stimulation mapping investigation. Neurosurgery 63:487-497, 2008

30. Luders E, Narr KL, Thompson PM, Woods RP, Rex DE, Jancke $\mathrm{L}$, et al: Mapping cortical gray matter in the young adult brain: effects of gender. Neuroimage 26:493-501, 2005

31. Martínez-Montes E, Valdés-Sosa PA, Miwakeichi F, Goldman RI, Cohen MS: Concurrent EEG/fMRI analysis by multiway partial least squares. Neuroimage 22:1023-1034, 2004

32. Mukamel R, Gelbard H, Arieli A, Hasson U, Fried I, Malach R: Coupling between neuronal firing, field potentials, and FMRI in human auditory cortex. Science 309:951-954, 2005

33. Narr KL, Bilder RM, Luders E, Thompson PM, Woods RP, Robinson D, et al: Asymmetries of cortical shape: effects of handedness, sex and schizophrenia. Neuroimage 34:939948, 2007

34. Ojemann G, Ojemann J, Lettich E, Berger M: Cortical language localization in left, dominant hemisphere. An electrical stimulation mapping investigation in 117 patients. J Neurosurg 71:316-326, 1989
35. Ojemann GA, Corina DP, Corrigan N, Schoenfield-McNeill J, Poliakov A, Zamora L, et al: Neuronal correlates of functional magnetic resonance imaging in human temporal cortex. Brain [epub ahead of print], 2009

36. Pignatti F, van den Bent M, Curran D, Debruyne C, Sylvester $\mathrm{R}$, Therasse $\mathrm{P}$, et al: Prognostic factors for survival in adult patients with cerebral low-grade glioma. J Clin Oncol 20: 2076-2084, 2002

37. Plaza M, Gatignol P, Leroy M, Duffau H: Speaking without Broca's area after tumor resection. Neurocase: 1-17 [epub ahead of print], 2009

38. Pouratian N, Bookheimer SY, O'Farrell AM, Sicotte NL, Cannestra AF, Becker D, et al: Optical imaging of bilingual cortical representations. Case report. J Neurosurg 93:676-681, 2000

39. Pouratian N, Bookheimer SY, Rex DE, Martin NA, Toga AW: Utility of preoperative functional magnetic resonance imaging for identifying language cortices in patients with vascular malformations. J Neurosurg 97:21-32, 2002

40. Pouratian N, Cannestra AF, Bookheimer SY, Martin NA, Toga AW: Variability of intraoperative electrocortical stimulation mapping parameters across and within individuals. J Neurosurg 101:458-466, 2004

41. Pouratian N, Sicotte N, Rex D, Martin NA, Becker D, Cannestra AF, et al: Spatial/temporal correlation of BOLD and optical intrinsic signals in humans. Magn Reson Med 47:766-776, 2002

42. Quiñones-Hinojosa A, Ojemann SG, Sanai N, Dillon WP, Berger MS: Preoperative correlation of intraoperative cortical mapping with magnetic resonance imaging landmarks to predict localization of the Broca area. J Neurosurg 99:311-318, 2003

43. Robles SG, Gatignol P, Lehéricy S, Duffau H: Long-term brain plasticity allowing a multistage surgical approach to World Health Organization Grade II gliomas in eloquent areas. J Neurosurg 109:615-624, 2008

44. Sanai N, Mirzadeh Z, Berger MS: Functional outcome after language mapping for glioma resection. N Engl J Med 358:18-27, 2008

45. Sanes JN, Donoghue JP, Thangaraj V, Edelman RR, Warach S: Shared neural substrates controlling hand movements in human motor cortex. Science 268:1775-1777, 1995

46. Schaltenbrand G, Wahren W: Atlas for Stereotaxy of the Human Brain. Stuttgart: Thieme Medical Publishers, 1998

47. Shattuck DW, Mirza M, Adisetiyo V, Hojatkashani C, Salamon G, Narr KL, et al: Construction of a 3D probabilistic atlas of human cortical structures. Neuroimage 39:1064-1080, 2008

48. Shinoura N, Suzuki Y, Yamada R, Tabei Y, Saito K, Yagi K: Precentral knob corresponds to the primary motor and premotor area. Can J Neurol Sci 36:227-233, 2009

49. Skirboll SS, Ojemann GA, Berger MS, Lettich E, Winn HR: Functional cortex and subcortical white matter located within gliomas. Neurosurgery 38:678-685, 1996

50. Smith JS, Chang EF, Lamborn KR, Chang SM, Prados MD, Cha $S$, et al: Role of extent of resection in the long-term outcome of low-grade hemispheric gliomas. J Clin Oncol 26:1338-1345, 2008

51. Sobottka SB, Bredow J, Beuthien-Baumann B, Reiss G, Schackert G, Steinmeier R: Comparison of functional brain PET images and intraoperative brain-mapping data using image-guided surgery. Comput Aided Surg 7:317-325, 2002

52. Spetzler RF, Martin NA: A proposed grading system for arteriovenous malformations. J Neurosurg 65:476-483, 1986

53. Steinmetz H, Volkmann J, Jäncke L, Freund HJ: Anatomical left-right asymmetry of language-related temporal cortex is different in left- and right-handers. Ann Neurol 29:315-319, 1991

54. Talairach J, Tournous P: Co-planar Stereotaxic Atlas of the Human Brain: 3-dimensional Proportional System-An 


\section{Reliability of neuroanatomy for prediction of eloquence}

Approach to Cererbral Imaging. New York: Thieme Medical Publishers, 1988

55. Tanriverdi T, Al-Jehani H, Poulin N, Olivier A: Functional results of electrical cortical stimulation of the lower sensory strip. J Clin Neurosci 16:1188-1194, 2009

56. Thompson PM, Moussai J, Zohoori S, Goldkorn A, Khan AA, Mega MS, et al: Cortical variability and asymmetry in normal aging and Alzheimer's disease. Cereb Cortex 8:492-509, 1998

57. Toga AW, Thompson PM, Mega MS, Narr KL, Blanton RE: Probabilistic approaches for atlasing normal and disease-specific brain variability. Anat Embryol (Berl) 204:267-282, 2001

58. Vikingstad EM, Cao Y, Thomas AJ, Johnson AF, Malik GM, Welch KM: Language hemispheric dominance in patients with congenital lesions of eloquent brain. Neurosurgery 47:562-570, 2000
59. Weisberg LA: The significance of nonvisualization of the cortical sulcal spaces on computed tomography. Comput Radiol 6:337-341, 1982

60. Yousry TA, Schmid UD, Alkadhi H, Schmidt D, Peraud A, Buettner A, et al: Localization of the motor hand area to a knob on the precentral gyrus. A new landmark. Brain 120:141-157, 1997

Manuscript submitted October 13, 2009.

Accepted November 10, 2009.

Address correspondence to: Nader Pouratian, M.D., Ph.D., Department of Neurosurgery, David Geffen School of Medicine at UCLA, Box 957049, Los Angeles, California 90095-7049. email: npouratian@mednet.ucla.edu. 